American Journal of Immunology 5 (1): 1-7, 2009

ISSN 1553-619X

(C) 2009 Science Publications

\title{
Clinical Evidence of Autologous Graft versus Tumor Effect
}

\author{
Luis F. Porrata \\ Department of Medicine, Division of Hematology, \\ Mayo Clinic College of Medicine, Rochester, MN, 55905
}

\begin{abstract}
Problem statement: The infused alloreactive lymphocytes in allogeneic stem cell transplantation (Allo-SCT) lead to an unspecific immune response causing Graft-Versus-Tumor (GVT) effect and Graft-Versus-Host Disease (GVHD). The current dogma regarding the anti-tumor effect of GVT is only observed in the Allo-SCT and not in the autologous hematopoietic stem cell transplantation (AHSCT). Approach: This article reviewed the medical literature to show clinical evidence of an autologous graft versus tumor effect. Results: Our group is the first one to publish that patients achieving a higher Absolute Lymphocyte Count (ALC), as a surrogate marker of immune recovery, recovery post-AHSCT experienced superior survival across multiple hematologic malignancies and solid tumors. Moreover, the ALC recovery post-AHSCT depended on the amount of infused ALC collected at the same time stem cells were collected in the autograft. Conclusion: The superior survival observed based on ALC recovery post-AHSCT provided the first clinical evidence of an autologous graft versus tumor effect. In addition, the association between the infused autograft ALC and ALC-recovery post-AHSCT suggested that the stem cell autograft should not be viewed only as the means to collect enough stem cells for hematologic engraftment, but also as an adoptive immunotherapeutic strategy to enhance immune recovery, transcelating into better clinical outcomes.
\end{abstract}

Key words: Absolute lymphocyte count; autologous graft versus tumor effect; autologous hematopoietic stem cell transplantation; survival; malignancies

\section{INTRODUCTION}

The adoptive transfer of donor immune effectors cells in Allogeneic Hematopoietic Stem Cell Transplantation (Allo-HSCT) has revived the interest in cellular immunotherapy of cancer as a potentially curative treatment modality ${ }^{[1]}$. The anti-tumor activity observed in the Allo-HSCT has been ascribed to the allogeneic Graft-Versus-Tumor (GVT) effect due to the infusion of alloreactive lymphocytes. The source of alloreactive lymphocytes is the donor stem cell inoculum of the allograft. In contrast, the high relapse rates observed after Autologous Hematopoietic Stem Cell Transplantation (AHSCT) are presumed in part due to the lack of $\mathrm{GVT}^{[2]}$. In this review, I will present clinical evidence of an autologous GVT mediated by the infusion of autologous lymphocytes.

Alloreactive graft versus tumor effect: The first preclinical evidence to suggest an immune-mediated GVT was reported in mice injected with leukemia and treated with total body irradiation ${ }^{[3]}$. Mice undergoing syngeneic stem cell transplantation died of leukemia recurrence, whereas mice receiving Allo-HSCT survived without evidence of leukemia, but developed
Graft-Versus-Host-Disease (GVHD). Mathe et al. ${ }^{[4]}$ coined the term "adoptive immunotherapy" for the antitumor effect of allogeneic cells. Allogeneic $\mathrm{T}$ and Natural Killer (NK) cells have been implicated in the development of GVT and GVHD ${ }^{[5-7]}$. The clinical evidence of an alloreactive GVT in humans has been attributed to: (1) Anecdotal reports demonstrating that abrupt withdrawal of immunosuppression in patients with tumor relapse post-allogeneic stem cell transplantation can re-establish complete remission, (2) Higher relapse rates after syngeneic transplant compared to Allo-HSCT, (3) The incidence of GVHD inversely correlates with the incidence of relapse after Allo-HSCT and (4) T-cell depletion of an Allo-HSCT increases the risk of relapse ${ }^{[1,8]}$. However, the most direct clinical evidence to support the anti-tumor activity of infused alloreactive lymphocytes comes from the immunotherapeutic strategy of Donor Lymphocyte Infusion (DLI) ${ }^{[9]}$.

Donor lymphocyte infusion: Donor Lymphocyte Infusion (DLI) provides the most direct clinical evidence of the anti-tumor effect of alloreactive lymphocytes. The North America and the European Bone Marrow Transplant (EBMT) reported that patients 
with chronic-phase Chronic Myelogenous Leukemia (CML) that relapsed after Allo-HSCT and were treated with DLI, in 76-79\% of cases complete cytogenetic and molecular remission were observed ${ }^{[10]}$. In appears for DLI to be most efficient patients have to be treated at the stage of minimal residual disease. DLI is most effective in slow growing tumors as only $20-30 \%$ responses have reported in acute myelogenous leukemia and only $15 \%$ in patients with rapidly progressing Acute Lymphoblastic Lymphoma (ALL) ${ }^{[11]}$.

Despite the potential clinical benefit of the adoptive GVT in Allo-HSCT, a fundamental problem is observed when using Allo-HSCT as an immunotherapeutic modality. The immune response observed after Allo-HSCT is not tumor specific, as the alloreactive lymphocytes that produce GVT also target the host (GVHD). To minimize the toxicity produced by GVHD and the high-dose chemotherapy in AlloHSCT, the concept of nonmyeloablative Allo-HSCT has been developed, where the hypothesis is that the adoptive GVT is enough to treat the underlying malignancy without the use of high-dose chemotherapy $^{[12]}$. The objective of chemotherapy/or radiation therapy in nonmyeloablative Allo-HSCT is primary directed at the production of an immunosuppressive state in the host, necessary to prevent rejection of the allo-graft. Thus, the TransplantRelated Mortality (TRM) in non-myeloablative AlloHSCT is believed to be reduced by minimizing the side effect from high-dose chemotherapy ${ }^{[13]}$.

DLI is used also in conjunction with nonmyeloablative Allo-HSCT to convert the host to a full donor chimerism. Unfortunately, the cumulative international data document a total incidence of clinically significant GVHD in at least $60 \%$ of patients treated with DLI. In addition, mild marrow dysfunction to severe and occasionally fatal marrow aplasia have been reported in $30 \%$ of patients treated with $\mathrm{DLI}^{[11]}$. Despite the GVT produced by alloreactive lymphocytes, it appears that TRM following AlloHSCT is mainly a toxicity of alloreactive lymphocytes rather than conditioning chemotherapy.

Lymphocyte recovery after stem cell transplantation: Post-Allo-HSCT studies have shown that early Absolute Lymphocyte Count (ALC) recovery, as a surrogate marker of immune reconstitution, is associated with prolonged survival $^{[14,15]}$. To assess whether early ALC recovery has prognostic significance post-ASCT, we analyzed the ALC at day 15 (ALC-15) post-ASCT in Multiple Myeloma (MM) and non-Hodgkin's lymphoma $(\mathrm{NHL})^{[16]}$. The median Overall Survival (OS) and
Progression-Free Survival (PFS) for the MM group were significantly better for patients with an ALC$15 \geq 500$ cells $\mu \mathrm{L}^{-1}$ versus an ALC- $15<500$ cells $\mu \mathrm{L}^{-1}$ (33 months Vs 12 months, $\mathrm{p}<0.0001$; 16 months Vs 8 months, $\mathrm{p}<0.0001$, respectively). In the NHL group, the median OS and PFS were also significantly better for patients with an ALC- $15 \geq 500$ cells $\mu \mathrm{L}^{-1}$ versus an ALC-15<500 cells $\mu \mathrm{L}^{-1}$ (not reached Vs 6 months, $\mathrm{p}<0.0001$; not reached Vs 4 months, p, 0.0001, respectively). Multivariate analysis demonstrated that ALC-15 was a powerful independent prognostic factor for OS and PFS in MM and NHL. We reported similar survival benefit with ALC-15 post-ASCT in Acute Myelogenous Leukemia (AML) ${ }^{[17]}$, Hodgkin's disease $^{[18]}$, metastatic breast cancer ${ }^{[19]}$ and primary systemic amyloidosis ${ }^{[20]}$. These findings have been confirmed by a number of investigators ${ }^{[21-23]}$. The lack of disease specificity suggests that the observed clinical outcomes may be related to successfully engrafted immune surveillance mechanisms reflected in the lymphocyte count post-ASCT. A limitation of all these studies is that all these studies have retrospective. We recently published a prospective study confirmed the finding of superior survival post-ASCT based on ALC15. The superior survival observed based on ALC recovery post-ASCT provides the first clinical evidence of an autologous graft-versus-tumor effect.

Timely of ALC recovery post-ASCT: A potential mechanistic explanation for the superior survival observed post-ASCT in patients achieving higher quantitative number of lymphocyte by day 15 is the concept of "tumor burden threshold" effect. In preclinical animal models, the dose of inoculated tumor cells affects the ability of the immune system to eradicate the tumor. In a syngeneic bone marrow transplant model, up-regulation post-transplant of the immune system with interleukin-2 (IL-2) in mice inoculated with either $10^{2}$ or $10^{4}$ B-cell leukemia/lymphoma (BCLI) cells at the same time as the transplant resulted in no tumor growth. However, no benefit was observed in mice receiving a BCLI challenge of $10^{6}$ cells $^{[24]}$. The timing of IL-2 therapy was also critical. Mice treated within one week of transplant did not survive due to disease progression. However, mice treated between 17 and 24 days posttransplant, where a rapid increase of peripheral mononuclear cells was observed, experienced prolonged survival. The authors argued that lymphocyte recovery is critical for the IL-2 administration in animals with minimal residual disease. Clinically, in the case of DLI/Allo-HSCT, patients with rapidly developing tumors (i.e., ALL) relapse following Allo-HSCT and 
DLI. One explanation for this observation is that tumor cells outgrow (and out-number) the engraft alloreactive donor lymphocytes outstripping their ability to generate the GVT effect ${ }^{[25]}$. Therefore, increased doses of DLI have been recommended in these cases ${ }^{[11,26]}$.

In addition to absolute quantitative recovery of the immune engraftment post-transplant, the timing of this process is also critical. A significant problem with DLI is the ongoing controversy of when best to infuse the donor lymphocyte to achieve the maximum immunotherapeutic effect. A significant problem with DLI is acute and chronic GVHD, which tend to be more severe if donor lymphocytes are infused earlier following Allo-HSCT ${ }^{[11]}$.

In the ASCT setting, we demonstrated superior OS and PFS in patients achieving a faster ALC recovery by day 15 compared to day 30 post-ASCT (not reached Vs 9 months, $\mathrm{p}<0.0001 ; 152$ months Vs 3 months, $\mathrm{p}<0.0001$, respectively) ${ }^{[27]}$. Thus, timely (early) quantitative reconstitution of lymphocytes (immune competence) post-ASCT is crucial for the eradication of minimal residual tumor in the host preventing tumor relapse.

Immunologic effector cells in early lymphocyte recovery post-ASCT: Having established ALC-15 as a surrogate marker of good clinical outcomes post-ASCT, we set out to understand which component (subset) of lymphocytes had the greatest impact on the observed effects. Natural Killer (NK) cells recover normal quantitative numbers and function by day 14 after ASCT compared to delayed quantitative and qualitative $\mathrm{T}$-and B-cells recovery after $\mathrm{ASCT}^{[28]}$. Moreover, NK cells are the first immune effector cells to recover after standard chemotherapy and Allo-HSCT, regardless of the underlying malignancy ${ }^{[2]}$. NK cells development can occur without a functional thymus in humans, perhaps allowing for the prompt NK cell recovery in adults ${ }^{[29,30]}$. In our prospective study, we showed that normal number of NK cells by day 15 was associated with superior OS and PFS (not reached Vs 5 months, $\mathrm{p}<0.001$; not reached Vs 3 months, $\mathrm{p}<0.0001$, respectively) ${ }^{[31]}$. These data suggest that early postASCT recovery of NK cells may have an important anti-tumor role in the putative autologous-GVT effect, as the only immune effector cells achieving normal numbers and function by two weeks post-ASCT.

Infused autograft lymphocytes as a source of lymphocyte recovery post-ASCT: The sources of lymphocyte recovery post-ASCT can be divided into two categories: (1) The host and (2) The stem cell autograft $^{[2]}$. From the host, lymphocyte sources include host stem cells and host lymphocytes surviving the high-dose chemotherapy. The host stem cells surviving high-dose chemotherapy most likely do not affect ALC15 post-ASCT because without stem cell graft support these patients remain myelosuppressed for prolonged period of time. To identify host lymphocytes surviving high-dose chemotherapy post-ASCT is difficult in comparison with Allo-HSCT where the development of mixed chimerism in Allo-HSCT allows discrimination of host Vs donor lymphocytes. Such discrimination is not possible in ASCT in the absence of marking studies of graft lymphocytes.

The second possible source of lymphocytes recovering post-ASCT is the autograft. From the autograft, lymphocytes could originate from: (1) Infused stem cells (CD34); or (2) Infused autograft lymphocytes $^{[2]}$. In our institution, as in many others, patients' autograft do not undergo any additional processing beyond cryopreservation, so that what is collected (CD34 \pm lymphocytes) is infused back to the patient. In other to understand the impact of the autograft on post-ASCT lymphocyte reconstitution, we set out to evaluate the impact of autograft CD34 and/or lymphocyte content on the kinetics of post-ASCT lymphocyte reconstitution. We identified no correlation between the amount of CD34 stem cells infused and ALC-15. However, a strong positive correlation was identified between the autograft lymphocyte content (autograft absolute lymphocyte count, A-ALC) and ALC-15. Patients that were infused with autografts containing higher A-ALC recovered greater numbers of lymphocytes by day 15 (ALC-15) and experienced significantly improved clinical outcomes in the setting of NHL and MM. We demonstrated superior OS and PFS in NHL and MM patients that were infused with an A-ALC $\geq 0.5 \times 10^{9}$ lymphocytes $\mathrm{kg}^{-1[32,33]}$. This finding has been recently confirmed by other investigators ${ }^{[34,35]}$. As expected, an inversed correlation was identified between the numbers of infused A-ALC and the time to achieve an $\mathrm{ALC} \geq 500$ cells $\mu \mathrm{L}^{-1}$ post-ASCT. Faster lymphocyte recovery post-ASCT was observed in patients with higher number of infused A-ALC ${ }^{[27]}$. These data suggest that the stem cell autograft in ASCT should not be viewed only for "bone marrow rescue" procedure to harvest CD34 stem cells necessary for hematologic engraftment, but also as an adoptive immunotherapeutic strategy in which autograft lymphocyte content directly influences tumor-related clinical outcomes in a number of different clinical settings ${ }^{[36]}$.

Autologous graft versus tumor effect: The association between A-ALC and ALC-15 provides the first clinical 
evidence of autologous graft-versus-tumor effect as the infusion of autograft lymphocytes has a direct impact not only on immune reconstitution, but also on survival post-ASCT, similar to the GVT observed in the AlloHSCT from the infused donor immune effector cells. In addition the infusion of autograft lymphocytes can be viewed as an autologous lymphocyte infusion similar to a donor lymphocyte infusion in the Allo-HSCT.

Ex vivo costimulation and expansion of $T$ lymphocytes for autologous lymphocyte infusion in ASCT: Recent studies have demonstrated ex vivo costimulation of T lymphocytes for ALI in ASCT can be feasible and with promising clinical activity. Before CD34 stem cell harvesting, patients underwent a single $20 \mathrm{~L}$ apheresis procedure that served as the source of cells for ex vivo expansion of $\mathrm{CD} 3+/ \mathrm{CD} 28+\mathrm{T}$ cells using anti-CD3 /anti-CD28 monoclonal antibodies coating micro beads. CD3+/CD28+ co-stimulated Tcells were infused back to the patients on day 14 postASCT. The authors reported rapid lymphocytosis with improved $\mathrm{CD} 3+$ and $\mathrm{CD} 4+\mathrm{T}$-cells and response to therapy in 16 patients with refractory relapse NHL, 32 patients with $\mathrm{MM}$ and in 4 patients with chronic myelogenous leukemia that were refractory to interferon- $\alpha$ and imatinib mesylate and no donor for Allo-HSCT prior to ASCT $^{[37-39]}$.

Autologous immunologic graft engineering: The process of peripheral blood stem cell collection involves two steps: (1) The use of the stem cell mobilization regimens to mobilize stem cells from the bone marrow into the peripheral blood in preparation for harvesting and (2) The autograft obtained by a combination of the apheresis machine and the number of collections to achieve the targeted stem cell count number. These two steps used to harvest enough stem cells for ASCT can be used to collect enough lymphocytes for ALI.

Stem cell mobilization: Peripheral Blood Stem Cell Collection (PBSC) is the clinically preferred stem cell modality over bone marrow harvest because of fewer procedure associated side-effects and faster hematopoietic engraftment ${ }^{[36]}$. The number of harvested stem cells (CD34 cells) depends on the ability to mobilize into the peripheral blood CD34+ cells from the bone marrow. Thus, the stem cell mobilization regimen that is able to produce the higher number of circulating peripheral blood CD34+ cells will translate in a high CD34+ yield collection. This same concept applies to the harvest of lymphocytes (A-ALC). The number of peripheral blood lymphocytes is directly proportional to higher A-ALC collection, leading to faster lymphocyte recovery post-ASCT ${ }^{[32,33,40]}$. Therefore, any intervention that might lead to precollection lymphopenia may harmfully affect postASCT lymphocyte recovery resulting in poor clinical outcomes. Our preliminary data suggest that MM patients mobilized with combination of chemotherapy (cyclophosphamide) and growth factor (G-CSF) resulted in lower of peripheral blood ALC leading to poor A-ALC collection compared to patients mobilized only with growth factor alone ${ }^{[36]}$. In spite of mobilizing higher number of $\mathrm{CD} 34^{+}$cells, the myelosuppressive effect of cyclophosphamide might be detrimental due to lymphopenia at the time of stem cell collection.

The combination of interleukin-2 (IL-2) and GCSF) has been used to mobilize immune effector cells (i.e., NK cells) in the autograft ${ }^{[36]}$. In a Phase I does escalation study of IL-2 following G-CSF administration during stem cell collection, we identified a dose of IL-2 that maximizes NK cells harvesting ${ }^{[36]}$. Other combinations of NK cell maturation and proliferation derived cytokines, such as interleukin-15 and interleukin-21 could be studied to assess their impact on autograft NK cell collection, NK cell recovery post-ASCT and clinical outcomes post-ASCT.

AMD3100 (Plexifor) is a reversible inhibitor of the binding of stromal derived factor (SDF-1) to its cognate receptor CXCR4. While AMD3100 mobilizes CD34+ cells by itself, it significantly enhances the mobilization capability of G-CF. AMD3100 has been developed mainly to maximize CD34+ cells collection ${ }^{[36]}$. However, in a control-matched study, we identified that patients mobilized with AMD3100 + G-CSF collected more lymphocytes than patients mobilized with G$\mathrm{CSF}^{[41]}$.

Apheresis collections and machines: The targeted CD34+ cell dose determines the number of apheresis collections. Different target doses are required for different clinical settings. Apheresis sessions continue until the requisite total CD34 count is achieved and the patient can proceed to conditioning chemotherapy. As described previously, CD34 content has no correlation with ALC-15 recovery post-ASCT nor does it impact on A-ALC content. Therefore, autograft adjustments to target CD34 dose will have no impact on A-ALC. Not surprisingly, we have described that in patients that require a greater number of apheresis sessions to achieve a required $\mathrm{CD} 34$ count, the total number of apheresed lymphocytes (A-ALC) are greater. In NHL patients that underwent $\geq 4$ apheresis collections experienced superior OS and PFS versus patients that did not ${ }^{[36]}$. Thus, as the number of apheresis collections 
help to achieve the targeted CD34 dose, the number of apheresis collections can be used to target an A-ALC dose with direct impact on clinical outcomes postASCT.

Likewise, apheresis instrument settings that have been optimized for CD34+cell collections may have different influences on overall A-ALC collections. We compared the A-ALC content of autograft collected by three different instruments (COBE Spectra, Fenwal CS 3000, Baxter Amicus) using manufacturer recommended settings. It was interesting to note that the different instruments harvested significantly different numbers of lymphocytes (A-ALC) ${ }^{[42]}$. The differences in A-ALC content impacted ALC-15 and survival in these patients. These data suggest that apheresis machines should be optimized to harvest more lymphocytes in addition to CD34+ cells as clinical outcomes appear to be directly impacted.

\section{CONCLUSION}

DLI in the Allo-HSCT setting provides the strongest clinical evidence of the potent anti-tumor effect of the immune system. Nevertheless, DLI does not circumvent the serious complications of GVHD. Conversely, ALI uses patients' own immune system to enhance existing anti-tumor defense mechanisms without the toxicity of GVHD. The focus of this review has been a discussion of new developments in ALI as means to improve clinical outcomes post-ASCT. The strategies can be divided into three categories. The first is to develop ex-vivo expansion of either T or NK cells to infuse post-ASCT. The second is to develop lymphocyte mobilization regimens in conjunction with stem cell mobilizations regimens to not only maximize CD34 stem cell mobilization into peripheral blood, but also immune effector cells (i.e., NK cells). The third is to modify the autograft collection process to collect a targeted A-ALC. We believe that rational strategies to create and deliver optimized ALI will translate as an effective potential to the treatment of malignant disease.

\section{REFERENCES}

1. Appelbaum, F.R., 2001. Hematopoietic cell transplantation as immunotherapy. Nature, 411: 385-389.

2. Porrata, L.F. and S.N. Markovic, 2004. Timely reconstitution of immune competence affects clinical outcome following autologous stem cell transplantation. Clin. Exp. Med., 4: 78-85. http://www.ncbi.nlm.nih.gov/pubmed/15672944
3. Barnes, D.W.H., M.J. Corp, J.F. Loutit and F.E. Neal, 1956 Treatment of murine leukemia with x-rays and homologous bone marrow. Preliminary communication. Br. Med. J., 2: 626-627. http://www.pubmedcentral.nih.gov/articlerender.fc gi?tool=pubmed \&pubmedid $=13356034$

4. Mathe, G., J.L. Amiel, L. Schwarzenberg, L. Cattan and M. Schneider, 1965. Adoptive immunotherapy of acute leukemias: Experimental and clinical results. Cancer Res., 25: 1525-1531. http://www.ncbi.nlm.nih.gov/pubmed/5323965

5. Ruggeri, L., M. Capanni and E. Urbani et al., 2002. Effectiveness of donor natural killer cell alloreactivity in mismatched hematopoietic transplants. Science, 295: 2097-2100 http://www.ncbi.nlm.nih.gov/pubmed/11896281.

6. Truitt, R.L. and A.A. Atasoyly, 1991. Contribution of CD4+ and CD8+ T cells to graft-versus-host and graft-versus-leukemia reactivity after transplantation of MHC-compatible bone marrow. Bone Marrow Transplantat., 8: 51-58. http://www.ncbi.nlm.nih.gov/pubmed/1833016

7. Palathumpat, V., S. Dejbakhsh-Jones and S. Strober, 1995. The role of purified CD8+ in graft-versusleukemia activity and engraftment after allogeneic bone marrow transplantation. Transplantation, 60: 355-361. http://www.ncbi.nlm.nih.gov/pubmed/7652765

8. Porter, D.L., 2001. The graft-versus-tumor potential of allogeneic cell therapy: An update on donor leukocyte infusions and non-myeloablative allogeneic stem cell transplantation. J. Hematother. Stem Cells Res., 10: 465-480. http://www.ncbi.nlm.nih.gov/pubmed/11522230

9. Slavin, S., 2001. Immunotherapy of cancer with alloreactive lymphocytes. Lancet, 2: 491-495. http://www.ncbi.nlm.nih.gov/pubmed/11905725

10. Kolb, H.J., A. Schattenberg, J.M. Goldman, B. Hertenstein and N. Jacobsen et al., 1995. Graftversus-leukemia effect of donor lymphocyte transfusions in marrow grafted patients. Blood, 86: 2041-2050. http://www.ncbi.nlm.nih.gov/pubmed/7655033

11. Slavin, S., S. Morecki, L. Weiss and R. Or, 2003. Immunotherapy of hematologic malignancies and metastatic solid tumors in experimental animals and man. Crit. Rev. Oncol./Hematol., 46: 139-163. http://www.ncbi.nlm.nih.gov/pubmed/12711359

12. Slavin, S., A. Nagler, E. Naparstek, Y. Kapelushnik and M. Aker et al., 1998. Nonmyeloablative stem cell transplantation and cell therapy as an alternative to conventional bone marrow transplantation with lethal cytoreduction for the treatment of malignant and nonmalignant hematological disease. Blood, 91: 756-763. http://www.ncbi.nlm.nih.gov/pubmed/9446633 
13. Hogan, W.J. and R. Storb, 2003. Therapeutic applications of non-myeloablative hematopoietic stem cell transplantation in malignant disease. Immunol. $\quad$ Res., 28: 1-11. http://www.ncbi.nlm.nih.gov/pubmed/12947220

14. Powles, R., S. Singhal, J. Treleaven, S. Kulkarni, C. Horton and J. Mehta, 1998. Identification of patients who may benefit from prophylactic immunotherapy after bone marrow transplantation for acute myeloid leukemia on the basis of lymphocyte recovery early after transplantation. Blood, 91: 3481-3486. http://bloodjournal.hematologylibrary.org/cgi/conte $\mathrm{nt} /$ short/91/9/3481

15. Pavletic, Z.S., S.S. Joshi, S.J. Pirruccello, S.R. Tarantolo and J. Kollalth et al., 1998. Lymphoid reconstitution after allogeneic blood stem cell transplantation for hematologic malignancies. Bone Marrow Transplant., 21: 33-41.

16. Porrata, L.F., M.A. Gertz, D.J. Inwards, M.R. Litzow and M.Q. Lacy et al., 2001. Early lymphocyte recovery predicts superior survival after autologous hematopoietic stem cell transplantation in multiple myeloma or non-Hodgkin lymphoma. Blood, 98: 579-585. http://cat.inist.fr/?aModele=afficheN\&cpsidt=1052912

17. Porrata, L.F., M.R. Litzow, A. Tefferi, L. Letendre and S. Kumar et al., 2002. Early lymphocyte recovery is a predictive factor for prolonged survival after autologous hematopoietic stem cell transplantation for acute myelogenous leukemia. Leukemia, 16: 1311-1318.

http://www.nature.com/leu/journal/v16/n7/full/240 2503a.html

18. Porrata, L.F., D.J. Inwards, I.N. Micallef, S.M. Ansell, S.M. Geyer and S.N. Markovic, 2002. Early lymphocyte recovery post-autologous haematopoietic stem cell transplantation is associated with better survival in Hodgkin's disease. Br. J. Haematol., 117: 629-633. http://www.ncbi.nlm.nih.gov/pubmed/12028034

19. Porrata, L.F., J.N. Ingle, M.R. Litzow, S.M. Geyer and S.N. Markovic, 2001. Prolonged survival associated with early lymphocyte recovery after autologous hematopoietic stem cell transplantation for patients with metastatic breast cancer. Bone Marrow Transplant. $\quad 28$ : 865-871. http://www.nature.com/bmt/journal/v28/n9/full/17 03236a.html

20. Porrata, G.M.A., M.R. Litzow, M.Q. Lacy, A. Dispenzieri and D.J. Inwards et al., 2005. Early lymphocyte recovery predicts superior survival after autologous hematopoietic stem cell transplantation for patients with primary systemic amyloidosis. Clin. Can. Res., 11: 1210-1218. http://cat.inist.fr/?aModele=afficheN\&cpsidt=16465932
21. Gordan, L.N., M.W. Surgue, J.W. Lynch and K.D. Williams et al., 2003. Correlation of early lymphocyte recovery and progression-free survival after autologous stem-cell transplant in patients with Hodgkin's and non-Hodgkin's lymphoma. Bone Marrow Transplant., 31: 1009-1013. DOI: 10.1038/sj.bmt. 1704050

22. Kim, H., H.J. Sohn, S.E. Kim, H.J. Kang, S. Park and S. Kim et al., 2004. Lymphocyte recovery as a positive predictor of prolonged survival after autologous peripheral blood stem cell transplantation in T-cell non-Hodgkin's lymphoma. Bone Marrow Transplant., 34: 43-49. http://cat.inist.fr/?aModele=afficheN\&cpsidt=15915790

23. Nieto, Y., E.J. Shpall, I.K. McNiece, S. Nawaz and J. Beaudet et al., 2004. Prognostic analysis of early lymphocyte recovery in patients with advanced breast cancer receiving high-dose chemotherapy with an autologous hematopoietic progenitor cell transplant. Clin. Can. Res., 10: 5076-5086. http://cat.inist.fr/?aModele=afficheN\&cpsidt=16014624

24. Ackerstein, A., E. Kedar and S. Slavin, 1991. Use of recombinant human interleukin-2 in conjunction with syngeneic bone marrow transplantation in mice as a model of control of minimal residual disease in malignant hematologic disorders. Blood, 78: 1212-1215. http://www.ncbi.nlm.nih.gov/pubmed/1878588

25. Mielcarck, M. and R. Storb, 2003. Nonmyeloablative hematopoietic cell transplantation as immunotherapy for hematopoietic malignancies. Cancer Treat. Rev., 29: 283-290. http://www.journals.elsevierhealth.com/periodicals /yctrv/article/PIIS0305737203000045/abstract

26. Peggs, K.S. and S. Mackinnon, 2001. Cellular therapy: Donor lymphocyte infusion. Curr. Opin. Hematol., $\quad$ 8: 349-354. http://www.ncbi.nlm.nih.gov/pubmed/11604574

27. Yoong, Y., L.F. Porrata, D.J. Inwards, S.M. Ansell and I.N. Micallef et al., 2005. The effect of absolute lymphocyte count recovery kinetics on survival after autologous stem cell transplantation for non-Hodgkin's lymphoma. Leukemia Lymphoma, 46: 1287-1294.

http://cat.inist.fr/?aModele $=$ afficheN\&cpsidt $=1704$ 3213

28. Porrata, L.F., D.J. Inwards, M.Q. Lacy and S.N. Markovic, 2001. Immunomodulation of early engrafted natural killer cells with interleukin-2 and interferon- $\alpha$ in autologous stem cell transplantation. Bone Marrow Trnasplant., 28: 673-680. http://www.nature.com/bmt/journal/v28/n7/abs/17032 03a.html 
29. Lotzova, E., C.A. Savary and R.E. Champlin, 1993. Genesis of human oncolytic natural killer cells from primitive CD34+CD33-bone marrow progenitors. J. Immunol., 150: 5263-5269. http://cat.inist.fr/?aModele $=$ afficheN\&cpsidt $=4832411$

30. Miller, J.S., C. Verfaillie and P. McGlave, 1992. The generation of human natural killer cells from CD34+/DR-primitive progenitors in long-term bone marrow culture. Blood, 80: 2182-2187. http://www.ncbi.nlm.nih.gov/pubmed/1384796

31. Porrata, L.F., D.J. Inwards, S.M. Ansell and I.N. Micallef et al., 2008. Early lymphocyte recovery predicts superior survival after autologous stem cell transplantation in non-Hodgkin lymphoma: A prospective study. Biol, Blood Marrow Transplant., 14: 807-816. DOI: 10.1016/j.bbmt.2008.04.013

32. Porrata, L.F., M.R. Litzow, D.J. Inwards and D.A. Gastineau et al., 2004. Infused peripheral blood autograft absolute lymphocyte count correlates with day 14 absolute lymphocyte count and clinical outcome after autologous peripheral blood stem cell transplantation in non-Hodgkin's lymphoma. Bone Marrow Transplant., 33: 291-298. DOI: $10.1038 /$ sj.bmt.1704355

33. Porrata, L.F., M.A. Gertz, S.M. Geyer, M.R. Litzow and D.A. Gastineau et al., 2004. The dose of infused lymphocytes in the autograft directly correlates with clinical outcomes after autologous peripheral blood hematopoietic stem cell transplantation in multiple myeloma. Leukemia, 18: 1085-1092. DOI: 10.1038/sj.leu.2403341

34. Symeonidis, A., S. Ings, N. Rabin, M. Watts and S. D'Sa et al., 2007. Patients with multiple myeloma, transplanted with lymphocyte-rich peripheral blood autografts have more favorable outcome. Haematologica, 92: PO-822. http://online.haematologica.org/Kos/browserecord. php?-action=browse \&-recid=411

35. Hiwase, D.K., S. Hiwase, M. Bailey, G. Bollard and A.P. Schwarer, 2008. Higher infused lymphocyte dose predicts higher lymphocyte recovery, which in turn, predicts superior overall survival following autologous hematopoietic stem cell transplantation for multiple myeloma. Biol. Blood Marrow Transplant., 14: 116-124. http://www.ncbi.nlm.nih.gov/pubmed/18158968

36. Porrata, L.F., M.R. Litzow and S.N. Markovic, 2005. Graft engineering for autologous stem cell transplantation. Gene Ther. Mol. Biol., 9: 121-134. http://www.gtmb.org/VOL9B/GTMBVOL6BPDF/ INDIVIDUAL/14._Porrata.pdf
37. Lapport, G.G., B.L. Levine, E.A. Stadtmauer and S.J. Schuster et al., 2003. Adoptive transfer of costimulated $\mathrm{T}$ cells induces lymphocytosis in patients with relapsed/refractory non-Hodgkin lymphoma following CD34+-selected hematopoietic cell transplantation. Blood, 102: 2004-2013. http://www.ncbi.nlm.nih.gov/pubmed/12763934

38. Rapoport, A.P., E.A. Stadtmauer, B.L. Levine, A. Badros and G. Akpek et al., 2004. Adoptive transfer of ex vivo costimulated autologous T-cells after autotransplantation for Myeloma (MM) accelerates post-transplant T-cell recovery. Biol. Blood Marrow, 10: 22-22. DOI: 10.1016/j.bbmt.2003.12.089

39. Rapoport, A.P., B.L. Levine, A. Badros, B. Meisenberg and K. Ruehle et al., 2004. Molecular remission of CML after autotransplantation followed by adoptive transfer of costimulated autologous $\mathrm{T}$ cells. Bone Marrow Transplant., 33: 53-60. http://www.ncbi.nlm.nih.gov/pubmed/14578928

40. Hiwase, D.K., S. Hiwase, M. bailey, G. Bollard and A.P. Schwarer, 2008. The role of stem cell mobilization regimen on lymphocyte collection yield in patients with multiple myeloma. Cytotherapy, $\quad$ 10: 507-517. http://www.ncbi.nlm.nih.gov/pubmed/18608354

41. Holtan, S.G., L.F. Porrata, I.N. Micallef, D.J. Padley and D.J. Inwards et al., 2007. AMD3100 affects autograft lymphocyte collection and progressionfree survival after autologous stem cell transplantation in non-Hodgkin lymphoma. Clin. Lymphoma Myeloma, 7: 315-318. http://www.ncbi.nlm.nih.gov/pubmed/17324341

42. Katipamula, R., L.F. Porrata, D.A. Gastineau, and Greiner C S.N. Markovic et al., 2005. Effect of apheresis instrumentation observed on survival following autologous hematopoietic stem cell transplantation for non-Hodgkin's. Bone Marrow Transplant., 37: 811-7. DOI: 10.1038/sj.bmt.1705338 